\title{
Standardising training in Neuroanaesthesia in India: Picking up the gauntlet
}

Neurosurgical anaesthesiology over the past decade has carved a unique and richly deserved niche for itself in the arena of medical sub-specialities. Technological refinements, better and accurate diagnostic modalities, monitoring advancements and in-depth research in areas which were hitherto beyond the realms of the broad speciality of anaesthesiology have propelled the growth of this sub-speciality by leaps and bounds.

Sub-speciality training is advantageous in that it prepares clinicians who are better experienced in particular areas and are, therefore, better equipped to deal with certain disorders. In addition, it ensures that certain essential standards of care are consistently met with while managing these disorders. Sub-speciality training generally attracts the highly talented individuals of the same speciality who are selected through rigorous entrance criteria and then undergo the meticulously designed training schedule. These factors finally culminate in enriching patient care and result in better patient outcomes.

Concurrent to its progress, a number of academic and professional courses, such as doctor of medicine, post-doctoral certificatecourseand post-doctoral fellowship (PDF), etc, providing training in the sub-speciality of neuroanaesthesiology, are being conducted by various institutes across India for anaesthesiology professionals. The specialised training is intended to produce highly skilled physicians who are better equipped to deliver neuroanaesthetic care in comparison to their counterparts from the anaesthesiology background.

However, maintaining similar standards of training and quality of learning across all these centres is currently a challenging task. Teaching and training in neuroanaesthesiology are influenced by the existing local practices, which lead to subtle differences in the protocols that are followed across different centres. In India, owing to its high population density and increased incidence of vehicular accidents, it contributes substantially to the neurosurgical patient population. In the face of considerable variations which exist in the teaching and training modalities of these courses in different centres, the need for development of a 'Standardised Training' is increasingly being felt and is the topic of heightened discussion.

A 'standard' is commonly accepted as a written policy which has been established by consensus and regulation based on proven or widely accepted principles. ${ }^{[1]}$ Regulations are drawn with regard to training and qualification of professionals dealing with operative procedures and perioperative care. Standardisation ensures uniformity of the quality of patient care, brings it at par with international standards and also leads to reduction in human errors, thereby increasing the overall patient safety. In the absence of standardised training, conflicting patterns of patient care cause clinical dilemmas whose resolution becomes difficult. In this context, the Indian Society of Neuroanaesthesiology and Critical Care (ISNACC) can play a stellar role and is the torchbearer of formulating the guiding principles which govern the training methodologies across the various institutes. The introduction of PDF courses being conducted at different centres across the country under the aegis of ISNACC is the foundation steps in this direction. Unlike the European and American courses, where the system of accreditation ensures that the necessary requirements for sub-speciality fellowships and training are being constantly monitored at the organisational levels by authorised professional bodies, we are yet to have such bodies. Another approach towards this direction can be the introduction of 'Credit Systems'. Worldwide, credit system is the watchword of numerous international training programmes. ${ }^{[2]}$ Credit-based certification systems ensure certain points for every level of learning and assessment throughout the duration the course. Thus, the achievement of standardised professional objectives is made certain based on the credits accumulated by the trainees.

Few measures can ensure standardisation of neuroanaesthesia in the country. First: Introduction of a common competitive examination for selection of candidates for admission to the sub-speciality training. Second: Drawing of a protocol-based curriculum for the trainees based on a consensus among the senior faculty members of different institutes imparting sub-speciality training. In case a particular procedure/surgery is not done in adequate numbers at a particular centre (the number to be recommended by the committee of senior members), the provision for liberal inter-centre student exchange should be available so that the candidates are exposed to wide varieties of cases/scenarios and no deficiencies in the teaching program exist. If necessary, provisions for academic grants for students to undergo observership in foreign centres should be 
made available. Point-based/credit-based evaluation should be encouraged where a minimum number of credit points must be accumulated by the students at the end of predetermined time periods. The points/credits should be awarded based on successful completion of or after attaining proficiency in specific teaching objectives. During the training period, a minimum number of research/scientific publications should be made mandatory to inculcate the habit of scientific writing in the trainees and they should be encouraged to carry out research activities. At the end of the tenure, a common exit examination for all the trainees should be conducted so that objectivity is maintained. In addition, in the case of veteran anaesthesiologists who are practising neuroanaesthesia without any specialised training, the ISNACC can play a leading role by organising teaching modules, bundles or CMEs at different locations under its banner for enhancement of their skills.

However, implementation of standardisation is not devoid of its share of problems. It entails the availability of sufficient number of trained neuroanaesthesiologists who are motivated to train their subordinates and at the same time constantly upgrade their competence levels to existing world standards. Increasing the existing burden on the available population of academic neuroanaesthesiologists (who are primarily responsible for training and availability of sufficient trainees which justifies the resource utilisation) is an added concern. The process of drawing up universally acceptable standards is a time-consuming job, and locally acceptable and feasible guidelines are a result of varying degrees of trials and errors. The basic structure of the locally designed training program of neuroanaesthesia or the core anaesthesiology curriculum has the potential to get disrupted following the enforcement of new standards. Moreover, application of credit system is subjected to integrity issues of work-based assessment and mentoring.

To conclude, certain logistical difficulties exist in implementation of a standardised curriculum for neuroanaesthesia training across the country. It would be a sensible approach for neuroanaesthesia mentors to envisage those roadblocks early and work proactively to formulate strategies for implementing standardised training program of neuroanaesthesia throughout the country. This will not only ensure universal professional competence beyond a minimum set standard among the trainees but will also immensely lift the standards of the sub-speciality. Therefore, picking up the gauntlet of standardising the training of neuroanaesthesia in the country by veterans of this field by amalgamating its dynamic changes and demands is the need of the hour.

\section{Shashi Srivastava, Rudrashish Haldar}

\author{
Department of Anaesthesiology, Sanjay Gandhi \\ Postgraduate Institute of Medical Sciences, \\ Lucknow, Uttar Pradesh, India \\ Address for correspondence: \\ Dr. Shashi Srivastava, \\ Department of Anaesthesiology, \\ Sanjay Gandhi Postgraduate Institute of Medical Sciences, \\ Lucknow - 226 014, Uttar Pradesh, India. \\ E-mail: shashian2002@yahoo.co.in
}

\section{REFERENCES}

1. Steiger HJ. Standards of neurosurgical procedures. Acta Neurochir Suppl 2001;78:89-92.

2. Raji JB, Velavan J, Anbarasi S, Grant L. Can credit systems help in family medicine training in developing countries? An innovative concept. J Family Med Prim Care 2014;3:183-7.

This is an open access article distributed under the terms of the Creative Commons Attribution-NonCommercial-ShareAlike 3.0 License, which allows others to remix, tweak, and build upon the work non-commercially, as long as the author is credited and the new creations are licensed under the identical terms.

\begin{tabular}{|l|l|}
\hline \multicolumn{2}{|c|}{ Access this article online } \\
\hline Quick Response Code: & Website: \\
\hline & www.jnaccjournal.org \\
\cline { 2 - 2 } & \\
\hline & \\
&
\end{tabular}

How to cite this article: Srivastava S, Haldar R. Standardising training in neuroanaesthesia in India: Picking up the gauntlet. $J$ Neuroanaesthesiol Crit Care 2016;3:195-6. 